\title{
Dos caras de la responsabilidad empresarial. Relaciones entre Celsia y la comunidad de El Caney durante la construcción de la central Hidromontañitas
}

\author{
Recibido: 21 de noviembre de 2016 \\ Aceptado: 24 de mayo de 2017 \\ Publicado: 27 de noviembre de 2017
}

\author{
Ángela Preciado Hoyos \\ angela.preciado@unisabana.edu.co \\ Universidad de La Sabana (Colombia)
}

Resumen: Este trabajo da cuenta de los aspectos más relevantes de la gestión de la responsabilidad empresarial y de las relaciones entre la empresa Celsia y un grupo de habitantes de El Caney (Santa Rosa de Osos, Colombia) durante la construcción de la central Hidromontañitas. La metodología seguida consistió en una entrevista en profundidad con la profesional de comunicaciones de Celsia y el desarrollo de un grupo de discusión con miembros de la comunidad. La información obtenida ha sido contrastada teniendo en cuenta qué entienden empresa y comunidad por responsabilidad empresarial, así como aspectos asociados al proceso de relación, en términos de participación de la comunidad, del cumplimiento de los compromisos pactados con ella, así como de las expectativas de esta última.

Palabras clave: Celsia, Hidromontañitas, responsabilidad empresarial, sector eléctrico colombiano.

\begin{abstract}
This paper gives an account of the most relevant aspects of the management of corporate responsibility and of the relations between the company Celsia and a group of inhabitants of El Caney in Santa Rosa de Osos, Colombia, during the construction of the Hidromontañitas plant. The methodology followed consisted of an in-depth interview with Celsia's communications professional and the development of a focus group with members of the community. The information obtained has been contrasted taking into account what companies and community understand about what is corporate responsibility, as well as aspects associated with the relationship process, in terms of community participation and the fulfilment of the commitments agreed with its expectations.
\end{abstract}

Key words: Celsia, Hidromontañitas, Corporate Responsibility, Colombian Electric Sector. 


\section{Introducción}

Si bien ha habido numerosas discusiones acerca de la dificultad de llegar a un concepto unificado sobre lo que es la responsabilidad social (Coppa y Sriramesh, 2013), suele haber acuerdo en torno al cumplimiento de una serie de condiciones por parte de las empresas para que sean responsables y que apuntan a ser eficientes en lo económico, respetando el medio ambiente y los derechos de las personas, sean estas empleadas, clientes, accionistas o comunidades (Vaaland y Heide, 2008), aportar al desarrollo de la sociedad en sus distintas vertientes (política, cultural, de desarrollo social), así como cumplir de manera ética con la legislación de cada país y con los pactos globales de protección de la naturaleza y de los derechos humanos (Luning, 2012).

Esta serie de condiciones relaciona la noción de triple balance contemplada en metodologías que se han desarrollado para guiar a las organizaciones en la elaboración de informes anuales en los que se reporta la responsabilidad empresarial, entre ellas el Global Reporting Initiative (GRI).

La responsabilidad empresarial ha sido definida, asimismo, como un compromiso que excede las obligaciones que han sido impuestas a las compañías, de manera implícita o explícita, por parte de la sociedad (Falck y Heblich, 2007). Es decir, lo ideal es que las actuaciones conducentes a responder a esta serie de cuestiones sean voluntarias (Clark, 2000; Vaaland y Heide, 2008; Recalde, 2010), por lo que cuentan las iniciativas que parten de la propia organización, sin que medien las presiones de gobiernos, organismos externos o grupos de activistas. En este contexto, ser responsable implica tomar decisiones desde el principio de precaución, después de examinar los riesgos asociados a ellas (Wirth y otros, 2016).

El elemento que diferencia la responsabilidad empresarial de conceptos como la filantropía, es que genera resultados sostenibles en el tiempo, por lo que debe ser planificada, supervisada y evaluada con regularidad. Algunos la ven como parte de una elección estratégica porque, al aplicarla, las organizaciones responden a prácticas corporativas que se inscriben en tendencias globales que aportan legitimidad a sus actuaciones (Jung y Kim, 2016).

Implantar la responsabilidad empresarial considerando las relaciones entre la empresa y la sociedad implica desarrollar y mantener relaciones con sus grupos de interés, pues no basta con la visión de la empresa para saber qué es lo que requiere el entorno, sino que hay que consultar al entorno mismo. En tal sentido, el ejercicio de la responsabilidad empresarial supone la comunicación permanente y fluida con actores diversos (Freeman y Phillips, 2002; Ihlen, 2008). El ajuste entre el desempeño de la empresa y los valores de quienes constituyen estos grupos es importante porque quienes no estén de acuerdo con las acciones de la entidad, pueden incidir en la ejecución de su estrategia corporativa (Vaaland y Heide, 2008; Coppa y Sriramesh, 2013).

Si se tiene en cuenta que cada grupo de interés tiene expectativas diversas de cara a las compañías, necesidades de información disímiles y respuestas diferenciadas frente a los canales de comunicación que están disponibles (Dawkins, 2004), es de esperar 
que la organización que busque ser responsable atienda de manera particular a cada uno de ellos.

Aunque la responsabilidad empresarial pudo percibirse en algún momento como un compromiso que se tenía de manera primordial con las comunidades, no abundan estudios académicos o publicaciones con resultados de investigaciones realizadas en Colombia, que den cuenta de este enfoque, ni siquiera desde una visión de comunicación empresarial. Tal circunstancia conduciría a afirmar que este es un ámbito novedoso para estudiar, incluso aunque su antigüedad pueda ser mayor en la práctica.

En la actualidad, se tiene conciencia de que la comunidad no es el único grupo de interés con el que las organizaciones deben ser responsables y se conocen esfuerzos por atender todo el espectro de stakeholders que tienen alguna relación con una entidad o que se ven afectados por ella (Moreno y Capriotti, 2007). Como se ha encontrado en el presente trabajo, convendría hacer más estudios en lo que atañe a cuestiones como el alcance que debe tener la responsabilidad de la empresa y cómo conjugarlo con las obligaciones correspondientes a otros actores sociales, entre ellos la propia comunidad y los entes públicos.

\subsection{Comunicación de la RSE}

Comunicar es requisito en los programas de responsabilidad empresarial para conocer los intereses y demandas de los grupos que inciden en las organizaciones, apoyar los procesos de negociación de expectativas de las diferentes partes (Clark, 2000; Self, 2010) y, en esa medida, hacer perceptibles los beneficios que se aportan a la sociedad, lo que incide de manera positiva en la gestión y el manejo de conflictos potenciales (Wirth y otros, 2016).

Por su naturaleza misma, la comunicación es un área de conocimiento que aporta una formación con énfasis en aspectos sociales, lo que permite afirmar que los profesionales formados en ella disponen de elementos para aportar en la relación con las comunidades (Leeper, 1996; Benn, Reiner y Pedleton, 2010). Campos como las relaciones públicas han buscado consolidar una base comunitaria para una práctica ética que habilite a las organizaciones a responder a su entorno de manera adecuada, en particular desde teorías que abogan por relaciones armoniosas entre la organización y su entorno (Grunig, 2011).

Según los postulados del movimiento comunitario (surgido en los noventas en los Estados Unidos), vivir en comunidad vincula una mejor relación con el propio sentido de responsabilidad personal y colectiva. A la par, obliga a establecer vínculos entre los derechos y responsabilidades que se adquieren al pertenecer a ella (Leeper, 1996). Desde esta mirada, se destaca que el verdadero sentido comunitario consiste en trabajar entre ciudadanos, para alcanzar retos en lo que tiene que ver con valores, hábitos y políticas públicas que conduzcan a la sociedad a salvaguardar y mejorar el futuro. De este modo, cuando una empresa se asienta en una región, entra a hacer parte de una comunidad específica y adquiere obligaciones con ella. 
En algunos estudios y teorías se habla de que, al trabajar en conjunto con otros actores sociales, la empresa adopta una ciudadanía corporativa, como sucedería con otros líderes y miembros que contribuyen con sus comunidades (Wood y Logsdon, 2008). Para alcanzar esa categoría, necesita comunicarse y orientar sus relaciones a crear colaboración con quienes hacen parte de su cadena de valor (Scandelius y Cohen, 2016). Pese a ello, lo que algunos han encontrado es que la comunicación ha sido el "vínculo ausente" en la práctica de la responsabilidad de las empresas con la sociedad (Chaudhri y Wang, 2007).

Las acciones de responsabilidad empresarial están interrelacionadas con la comunicación, además de otras razones, porque la responsabilidad empresarial es una manera de comportarse. Las actuaciones son una forma de expresión mucho más clara y contundente que otras formas discursivas. Aparte de ello, uno de los aspectos que revela el interés de la organización por su entorno es el grado en el que se abre al escuchar a los agentes que están en él y la medida en que llega a reflejar esos acuerdos en sus decisiones y hechos. Si los agentes que se comunican con la empresa no sienten que el diálogo que mantienen con ella surta algún efecto, más pronto que tarde dejarán de acercársele y se generará falta de confianza y credibilidad.

En estudios realizados sobre la participación de los colombianos en procesos de construcción de comunidad y tejido social, se destaca que la confianza es el primer requisito para que pueda haber participación (Rodríguez-Raga y Seligson, 2010). Según estos trabajos, las personas que sienten mayor confianza pueden tener más incentivos para participar en instancias de la sociedad civil y en otras más institucionalizadas. De igual modo, quien tiene experiencias satisfactorias en ejercicios de participación puede empezar a confiar más en sus conciudadanos. En los mismos estudios se ha encontrado que el porcentaje de colombianos que participa al menos una vez al año en algún grupo para resolver un problema de la comunidad, es del $30 \%$, índice que figura entre los menores del continente, lejos de las proporciones de Estados Unidos. En estos estudios el nivel más bajo de participación de los colombianos se presenta en lo que atañe a juntas o comités de mejoras para la comunidad (Rodríguez-Raga y Seligson, 2010).

El propósito de la investigación que aquí se presenta ha sido conocer la manera como se ha establecido la relación entre Celsia y la comunidad de El Caney, población localizada en Santa Rosa de Osos (Departamento de Antioquia), en el proceso de construcción de la central Hidromontañitas. Se plantea como hipótesis que si la empresa generó un proceso de comunicación transparente y abierta, en el que hubo participación de los miembros de la comunidad, esta tendrá una visión positiva y de confianza sobre Celsia, lo que no es posible que suceda si dicho proceso no ha sido claro e incluyente. En los siguientes apartes de este artículo se presentan la metodología, los resultados obtenidos, lo mismo que las conclusiones a las que se han llegado.

\subsection{Celsia}

Celsia es una empresa de origen colombiano. Se especializa en proyectos de generación de energía hidráulica y térmica. Es una de las tres mayores empresas de generación de energía 
en Colombia, junto con EPM e Isagen y socia mayoritaria de la Empresa de Energía del Pacífico (EPSA) que a su vez maneja tres negocios: generación, distribución y transmisión de energía. Su principal accionista es el Grupo Argos y tiene un capital 100\% privado. Si bien es una compañía con diez años de historia, tiene más de 90 de tradición, por cuanto sus orígenes se remontan a Coltabaco.

Celsia surgió de la escisión del negocio del tabaco y las inversiones que la misma empresa manejaba y que durante algunos años se amparó bajo el nombre de Colinversiones. A partir de un proyecto de re direccionamiento estratégico, creció y se especializó en el negocio de la energía, al que se dedica en la actualidad. Debido a este cambio de orientación, buscó renovar su identidad y eligió un nombre que, a juicio de la compañía, refleja el resultado obtenido con la transformación estratégica y operativa que tuvo lugar. Fue así como optó por llamarse Celsia a partir de abril de 2012. Ese mismo año produjo $5.038 \mathrm{GWh}$., lo que equivale al $8.4 \%$ de la demanda total de energía del mercado colombiano (Celsia, 2013).

Hidromontañistas es una de las centrales que Celsia opera en la actualidad. Los trabajos para construirla empezaron en febrero de 2009 y la central entró a operar en junio de 2012 . La inversión que se hizo en la obra fue de \$127.000 millones. De esa cantidad, la empresa destinó \$7.200 millones a la inversión social, medio ambiental, y a la mejora de vías. En la etapa de construcción de las obras, la empresa debía destinar el 1\% del presupuesto total a estas actividades, pero invirtió el 5,6\%. Es decir, más de lo que demandaban las leyes.

Una vez concluida la obra, la empresa, como todas las del sector, ha debido acogerse al artículo 45 de la Ley 99 de 1993 y a su decreto reglamentario No. 1993 de 1994 de la República de Colombia, sobre las transferencias por venta de energía. Estas consisten en dineros que deben entregar a los municipios localizados en el área de la cuenca hidrográfica que aporta a los embalses y aledaños a las zonas inundadas por estos, lo mismo que a las Corporaciones Autónomas Regionales ${ }^{1}$.

\section{Objetivos}

El objetivo de la presente investigación ha consistido en determinar la visión que empresa y comunidad tienen sobre lo que es la responsabilidad empresarial y el apoyo que la comunicación ha brindado en el caso que se analiza. Para averiguarlo, el trabajo

1. Las transferencias representan el $6 \%$ de las ventas brutas de energía y son distribuidas así: 3\% a las corporaciones autónomas regionales del área donde están ubicados la cuenca hidrográfica y el embalse; 3\% para los municipios localizados en la cuenca hidrográfica, donde el 1,5\% es para los municipios que surten el embalse y 1,5\% para los municipios donde está ubicado el embalse. En los municipios, las transferencias sólo pueden ser utilizadas en la ejecución de obras que se encuentren en el Plan de Desarrollo Municipal con prioridad en los proyectos de saneamiento básico y mejora ambiental, como alcantarillados, tratamiento de aguas, manejo y disposición de desechos sólidos y líquidos, cuidado de bosques y suelo, reforestación, biodiversidad, calidad de vida urbana, producción más limpia, procesos de productos endógenos, mercados verdes, educación ambiental (Empresa Urrá, 2012). 
se orientó a buscar respuesta a las siguientes cuestiones: a) qué entiende la empresa por responsabilidad social empresarial y qué entiende la comunidad; $b$ ) qué acciones emprende la organización desde el concepto de responsabilidad que maneja y cuáles de esas acciones se ajustan a un comportamiento responsable con los miembros de la comunidad; $c$ ) en qué medida la empresa buscó involucrar a la comunidad de El Caney en el proceso de construcción de la central hidroeléctrica y, de igual modo, d) cuáles fueron los aportes de la comunidad en él.

\section{Metodología}

Para conocer la visión de Celsia se entrevistó a la persona responsable del área de Comunicaciones, por ser quien tenía el conocimiento de la estrategia que guio el proceso de relación y comunicación, a la vez que conocía en detalle la orientación filosófica de la empresa en relación con la responsabilidad empresarial. Su cargo se denominaba "Profesional de comunicaciones". En el momento en el que se hizo el estudio, se ocupaba de todas las actividades del área en la organización. La entrevista se realizó durante el mes de marzo de 2012 en las oficinas de Celsia, ubicadas en Medellín. Esta entrevista y la lectura de los informes de gestión de 2011 permitieron conocer el enfoque que Celsia manejaba para relacionarse con la comunidad. El cuestionario (a partir del cual se orientó la conversación) incluía veinte preguntas semiestructuradas, que permitieron indagar sobre asuntos puntuales y posibilitaron preguntar por aspectos complementarios para que la entrevistada explicara con detalle respuestas que merecían ser ampliadas.

La entrevista se diseñó en tres partes. En la primera se consultó por el enfoque que la organización eligió para gestionar su responsabilidad. En un segundo segmento se preguntó por la gestión que la empresa hace de la responsabilidad social entre los grupos de interés y, en particular, entre las comunidades. El tercer y último fragmento se centró en preguntas sobre la manera en que la comunicación apoya los programas de responsabilidad empresarial que se desarrollan con estos grupos.

Para indagar por los mismos temas entre la comunidad, se consideró que la metodología más indicada era la sesión grupal o grupo de discusión, porque este permite conocer ideas, opiniones o actitudes colectivas; en este caso, de las personas que habitan zonas aledañas a las áreas de operación de la entidad. Del mismo modo, ayuda a percibir la dinámica de las relaciones entre los individuos y el sentido que ellos generan a partir de sus conversaciones, por cuanto "las hablas individuales tratan de acoplarse al sentido social [y] la reordenación del sentido social requiere de la interacción comunicativa" (Galeano, 2009: 191). Es decir, la metodología permitía recoger el pensar de diferentes miembros de la comunidad.

La convocatoria a la sesión grupal se hizo por intermedio de la Secretaría de Desarrollo Comunitario del municipio de Santa Rosa de Osos. Se pidió, como condición, que los participantes fueran mayores de edad, habitantes del sector y un número no mayor a 10 personas, de manera que todos pudieran intervenir en la conversación. Un promotor de desarrollo comunitario contactó y comprometió a los habitantes de la vereda para la fecha prevista. 
En el grupo de discusión participaron nueve personas, seis mujeres y tres hombres. Con excepción de una de las mujeres (que trabaja en una fábrica de golosinas) las demás se dedican a labores del hogar. En cuanto a los hombres, uno es jubilado, el otro atiende un negocio familiar de venta de comestibles junto con su esposa, y el otro no tiene un trabajo permanente. La reunión se hizo en el único salón que tiene la escuela de la vereda y tuvo una duración de dos horas.

Se pidió permiso a los asistentes para grabar las respuestas en audio. La persona que moderó el grupo focal fue la investigadora principal del proyecto, a partir del cual se ha generado el presente artículo. La misma persona hizo la entrevista a la profesional de Comunicaciones de Celsia. Como moderadora, se orientó a buscar la intervención de todos los asistentes y a profundizar en puntos de vista dispares para conocer razones, motivos, y para no dar por sentada una sola posición. Algunos participantes manifestaban sus opiniones, pero no profundizaban en ellas, por lo que se les pedía explicarlas con detalle para comprender mejor el sentido al que apuntaban.

La guía de preguntas del grupo de discusión se diseñó en el siguiente orden temático: $a$ ) presentación de los propósitos de la reunión, de la manera como cada uno podía participar, de la persona que actuaba como moderadora en la discusión y de los participantes; $b$ ) conversación inicial en torno a la idea que los participantes tenían sobre lo que es la responsabilidad, en general, y la responsabilidad de las empresas, en particular; $c$ ) conocimiento que se tenía sobre Celsia y medida en la que se consideraba que ha sido una empresa que se pudiera catalogar como responsable; $d$ ) acciones de responsabilidad social de la empresa que recuerden que se hayan desarrollado durante el tiempo de construcción de la central hidroeléctrica y e) manera como se dio la relación entre ellos y la empresa.

El cuestionario que se dirigió a la profesional de comunicaciones de Celsia, lo mismo que la guía de preguntas del grupo de discusión, fueron probados y revisados de manera previa por investigadores que hacen parte del Centro de Investigaciones de la Comunicación Corporativa y Organizacional, Cicco, de la Universidad de La Sabana, en el que se inscribió el proyecto del que se derivó el presente trabajo.

\section{Resultados}

\subsection{Conceptos de responsabilidad empresarial}

En cuanto al concepto de responsabilidad empresarial que manejan las partes, Celsia ha optado por abordarlo desde la denominación "desarrollo sostenible", porque para ellos abarca (además de lo social) lo económico, lo ambiental y, como perspectiva, puede gestionarse de una manera más transversal a toda la organización. Para la profesional de comunicaciones, verlo de esa manera hace que no se agote en el relacionamiento con el grupo de interés comunidad, que es como se llegó a entender, en algún momento, la noción de "responsabilidad social" e imprime un alcance mucho mayor a la gestión de asuntos asociados. 
El área que se encarga de gestionar estos aspectos en la estructura organizacional recibe el mismo nombre. A ella está adscrita la Fundación Celsia, "que más que entregar aportes, soporta proyectos que garantizan que las comunidades sean auto sostenibles" (Isagen, 2012). Las temáticas que más interesa apoyar son cultura y medio ambiente.

En cuanto al punto de vista de los habitantes de El Caney, ser responsable implica "cumplir los compromisos que uno hace con otros", es ese "algo" que uno adquiere cuando establece compromisos. "Un asunto de dos partes", porque "si uno es responsable con uno mismo, puede serlo con la comunidad, con el lugar donde trabaja y con el trabajo que hace". En consecuencia, "implica que lo que estamos haciendo tenga buenos resultados".

Como parte del desarrollo del grupo de discusión se pregunta si, ateniéndose al significado que ellos adjudican a la responsabilidad, una empresa puede serlo. Comentan que sucede cuando paga los sueldos a tiempo, cuando sus productos son de buena calidad y cuando cumple las promesas que hace a los demás. A este último aspecto otorgan el mayor significado.

Se plantea a continuación si, atendiendo a las reflexiones previas, la empresa Celsia es responsable. Es preciso hacer notar que los participantes en el grupo de discusión no reconocían en ese momento el nombre de Celsia. Para ellos, la empresa se seguía llamando "Colinversiones". La empresa hizo el anuncio del cambio de nombre tres meses atrás. Algunos de los miembros de la comunidad llegaron a afirmar que se trataba de dos organizaciones: "Colinversiones", que construyó la central, y "Celsia", que a partir de ese momento se iba a encargar de operarla.

Uno de los asistentes dijo recordar que en una misa de domingo el sacerdote informó sobre el cambio de nombre. Una vez hecha la aclaración, se encontró que las opiniones acerca de si Celsia era o no una empresa responsable, estaban divididas. La razón central se hallaba en que, para algunos, había cumplido con los acuerdos pactados, pero para otros, todavía había algún compromiso pendiente.

El siguiente tema en la conversación consistió en determinar cuáles fueron los compromisos pactados, cuáles los verificados y cuáles quedaban por cumplir, desde el punto de vista de los asistentes. Para contrastar esta serie de respuestas, se consultó con la profesional de comunicaciones por las realizaciones de la empresa en la zona de influencia, teniendo en cuenta que estas reflejan la manera en que se materializa la visión que tiene Celsia sobre su responsabilidad y las cuestiones en las que hace énfasis.

\subsection{Acciones de responsabilidad empresarial}

Como explica la profesional de comunicaciones, las actividades que la empresa desarrolló en los dos últimos años con las comunidades aledañas a la central Hidromontañitas, incluyeron la formación de personas de la comunidad en tecnologías orientadas a la conservación y preservación del medio ambiente. Para ello (y junto con los habitantes del sector) definieron cinco tecnologías que estos últimos, por decisión personal, quisieran 
aprovechar en sus predios. Estas tenían que ver con semi-invernaderos, fogones eficientes, filtros de agua o porci-cultivos. Todas ellas se dieron a conocer en talleres participativos, después de los cuales se entregó una cartilla en la que se contaba, paso a paso, cómo construirlas para que pudiera implementarlas todo aquel que quisiera.

Otro aporte que se hizo fue la dotación de un refrigerador y una nevera industrial para la fábrica de golosinas, con el fin de apoyar el fortalecimiento de una de las principales organizaciones comunitarias de la región. De esta manera, la empresa buscaba cumplir su propósito de apoyar proyectos auto sostenibles para formar núcleos productores, ajustados a las demandas del mercado (Coliversiones, 2012).

En materia de educación se hicieron reparaciones en el centro educativo rural (Escuela de El Caney), las cuales abarcaron arreglos en el piso, las puertas y el techo. Se instalaron muebles, estanterías, así como dos juegos infantiles; se repartieron kits escolares y se ofrecieron clases de inglés; se hicieron adecuaciones en la capilla y en la carretera de acceso a la vereda, que es la misma vía para llegar a la central hidroeléctrica. Para Celsia, apoyar a la comunidad en estos aspectos les permitía, como empresa, cumplir con el propósito de fortalecer la capacidad de gestión de la comunidad, ajustados a sus características económicas, culturales, políticas y sociales (Colinversiones, 2012).

Cuando se consulta a los miembros de la comunidad por las acciones de responsabilidad empresarial que ejecutó Celsia y que hacían parte de los acuerdos pactados, citan las obras hechas en la escuela y la capilla, lo mismo que los programas y talleres orientados a la conservación del medio ambiente. No citan por ellos mismos la entrega de los kits escolares, ni mencionan los equipos entregados a la fábrica de golosinas, pese a que como se ha dicho, allí trabaja una de las asistentes al grupo de discusión.

Algunos participantes del grupo insisten en que, a pesar de que recibieron numerosos beneficios (empleo, brigadas de salud, medicinas), la empresa no cumplió con el mantenimiento de la vía de acceso ni con la recolección de basuras. En cuanto al primer tema, una persona afirma que "mantuvieron la vía en perfecto estado mientras estuvieron aquí". Otra asegura que prometieron pavimentarla, en tanto otros asistentes comentan que "ellos sólo se comprometieron con el afirmado del suelo".

Entre la totalidad de acciones citadas, el principal desacuerdo que se presenta entre los integrantes del grupo ha sido el de la vía, asunto que ha generado el mayor reparo en lo que tiene que ver con compromisos que se pactaron con la empresa. Cuando se pregunta si la vía está mejor o peor, no hay convergencia en las opiniones. La persona que tiene la percepción más positiva sobre el proceso asegura que "el hecho de que la comunidad a veces no responda, no es culpa de la empresa". A su juicio, Celsia consiguió convocar a adultos y niños, hizo que muchas personas participaran en actividades colectivas que de otro modo ni ellos como comunidad, ni otros agentes, hubieran desarrollado.

En contraposición, está quien afirma que siempre se presentan las mismas personas a las reuniones en las que se cita a la comunidad, porque "la gente está cansada de estar 
viniendo para no ver el resultado. Venimos en la medida en que hay resultados. Aquí estamos cansados de que la administración y todos nos prometan un poco de cosas y no cumplan". Alguien interviene para decir que Celsia debería comprometerse con un carro recolector de basura que pase cada ocho días. Se pregunta si el municipio de Santa Rosa de Osos se ocupa de esta labor, por tratarse de un servicio público, a lo que algunos responden que por allí no pasa el carro de la basura y se genera mucha, por tratarse de una zona turística.

Un miembro del grupo considera que tal vez no debería ser una obligación de la empresa, pero como lo estuvo haciendo durante un tiempo, podría prolongar el compromiso o compartir la responsabilidad con las empresas públicas: "Es verdad que ellos estuvieron gestionando eso con la alcaldía, pero el alcalde que había no nos pudo cumplir con todo lo que dijo [...] y es que ahora, como ellos (Celsia) están aquí, el municipio de Santa Rosa no nos aporta nada...". Sobre la gestión de los desperdicios, uno de los asistentes hace notar que la empresa siempre tuvo la posición de no quemarlos y les motivaba, más bien, a reciclar.

La conversación conduce a formular dos preguntas: ¿hasta cuándo consideran ellos que la empresa debe recoger las basuras y arreglar la vía? Y, en segundo término, ¿qué han aportado ellos, como comunidad, a este proceso? En el primer aspecto, algunas personas consideran que la empresa debe seguir trabajando con ellos y con la alcaldía; en el segundo, que ellos aportaron tiempo en las reuniones y, en algunos casos, mano de obra.

Entre los asistentes no se tiene un conocimiento claro sobre la legislación que cobija a las empresas generadoras de energía, en cuanto a que en el momento en el que entra a operar la central, los aportes a la zona de influencia se hacen a partir de las transferencias, que como se ha señalado en líneas anteriores, se entregan a las administraciones municipales y a las corporaciones autónomas regionales y son recursos destinados, por ley, a asuntos ambientales.

Estas empresas tienen la alternativa de destinar recursos, una vez concluidas las obras, a programas de inversión social. En esta instancia la inversión es voluntaria, lo mismo que las decisiones en torno de los temas en los que se incidirá. Este aspecto no ha sido objeto de evaluación en el presente trabajo, debido a que los instrumentos de investigación se aplicaron, como se ha advertido, en una etapa intermedia: entre la finalización de las obras de infraestructura y el momento en el que iba a empezar a generarse energía.

\subsection{Comunicación y proceso de relaciones}

Para identificar los grupos de interés más relevantes en su gestión, Celsia se ha basado en determinar el impacto de sus acciones, en el nivel de participación que pueden tener en la organización y en los riesgos y oportunidades que la gestión de la empresa pueda ocasionar. Con esta información construyó su filosofía de actuación con cada uno de sus grupos de interés (Celsia, 2013). 
Los medios de relacionamiento que la empresa tiene estipulados con la comunidad son reuniones, talleres de trabajo participativo, una oficina de atención, convocatorias públicas y proyectos de inversión social. Como se aprecia en el caso de El Caney, el proceso de relacionamiento con los miembros de la comunidad es determinante en la valoración que ellos hacen sobre el grado en el que la empresa ha sido responsable o no, porque si no quedaron claros los compromisos en la primera etapa de negociación de intereses, es probable que (al pasar el tiempo) se juzgue a la empresa como incumplida.

Otra de las preguntas que se hizo en el grupo de discusión ha sido si la comunidad fue consultada sobre la pertinencia de los proyectos desarrollados. Lo que se encontró es que no existe un punto de vista unificado porque cada persona lo valora de acuerdo con el grado en el que se involucró en el proceso y eso no depende de la empresa, sino del interés y voluntad de cada uno.

En febrero de 2009, antes de iniciar las obras, Celsia reunió a la comunidad asentada en la zona de influencia de Hidromontañitas para que todos tuvieran conocimiento sobre lo que se iba a hacer y les informó sobre las diferentes situaciones que podían presentarse como consecuencia del desarrollo de las obras. Una de las participantes en la reunión asegura que, teniendo en cuenta el panorama que les presentaron en ese momento, fue poco, en realidad, lo que tuvieron que enfrentar. El grupo está de acuerdo en que hubo casos de vicio (como denominan el consumo de drogas), en contratistas venidos de fuera que, por fortuna, no contagiaron a jóvenes, niños o personas de la comunidad.

Las convocatorias a las reuniones en las que se generaron los acuerdos se hicieron por intermediación de los líderes y las juntas de acción comunal. De parte de Celsia, la relación estuvo en cabeza de una ingeniera, a quien los miembros de la comunidad consideran portavoz de la empresa en la etapa de construcción de la central. En Celsia coinciden en que esa es la persona que estuvo llevando la relación directa con los habitantes de la zona.

La profesional de comunicaciones de Celsia comenta que, hasta el momento en el que se hizo la entrevista para el presente estudio, no había un profesional especializado, encargado de apoyar la comunicación con las comunidades. Este iba a contratarse por esos días, en parte, porque la empresa había ganado una licitación para una nueva obra, Porvenir II. La llegada del comunicador sólo hasta ese momento se explica de este modo: "Hemos ido creciendo muy aceleradamente [por eso] viene un comunicador que se va a encargar de la comunicación con comunidades. Ha trabajado con Porce III, de EPM y con Hidroituango (proyectos de generación de otra empresa de energía colombiana). Es una persona con mucha experiencia”.

Sobre la relevancia de la comunicación en el proceso, la entrevistada reflexiona de la siguiente manera: "Esto llama mucho la atención, porque tú encuentras en las comunidades, siempre unas frases que son muy constantes cuando una compañía de estas llega a construir un proyecto hidroeléctrico. Casi creen que son los que los van a sacar de todos los problemas o las necesidades que tienen, pero hay unos límites...". La comunicación, en este caso, sería un medio para que las comunidades tomen conciencia de los compromisos y metas que puede llegar a tener cada uno de los actores que incide en su entorno y en su estabilidad. 
Sobre los aportes que hace la comunicación al proceso, la profesional de comunicaciones explica:

\begin{abstract}
"Tenemos un plan de comunicaciones y en ese plan demarcamos unos grupos de interés. Entre ellos está la comunidad. De la mano del área de Desarrollo sostenible miramos cómo son esas actividades y cómo las debemos apoyar. Si se necesita crear nuevos medios, si se requiere difundir la información de otra manera, entonces, por ejemplo, encontramos que era muy importante tener una comunicación directa con la comunidad, para apuntarle, en el caso de Hidromontañitas, a divulgar cómo se estaba desarrollando el proyecto".
\end{abstract}

Por eso crearon el periódico "Nuestra energía", que se publica cada tres meses. "Allí contamos cómo se desarrolla el proyecto, cuáles son las actividades que realizamos, además porque a la comunidad le gusta mucho verse ahí reflejada". Al consultar con la comunidad, se verifica que este medio es muy bien recordado, aunque no encierre, en sí mismo, una forma de comunicación personalizada.

Como parte de la primera etapa del proyecto, se hizo una reunión a la que asistieron el presidente de la compañía y los alcaldes de los municipios de Don Matías y Santa Rosa de Osos. Les presentaron la compañía, lo mismo que el proyecto. "Obviamente las administraciones dijeron: qué tan bueno [...] nos alegra mucho, esperamos que esto represente, también para la comunidad, un desarrollo y un bienestar, y [en esa reunión] ellos manifestaron que querían apoyo para unos y otros proyectos" (entrevista personal con la profesional de comunicaciones de Celsia, 2012).

Estas solicitudes siguieron llegando durante la etapa de construcción de la central y se canalizaron por intermedio del director del proyecto. Si el tema involucrado en la petición hacía parte de sus funciones, él tomaba las decisiones del caso. Si no, escalaba las solicitudes a la presidencia y ésta definía si podía hacerse o no.

\title{
5. Conclusiones
}

El concepto de sostenibilidad adoptado por Celsia (para cobijar la filosofía, la gestión y las diferentes acciones de responsabilidad empresarial que realiza) enlaza una condición que se ha venido señalando como necesaria en este ámbito y es el desarrollo de programas que tengan impacto en el largo plazo. Se percibe esta intención en las acciones formativas y en el apoyo a proyectos productivos en la zona.

Es evidente que en la comunidad se generan muchas expectativas con la llegada de una empresa de este tipo a su región, en parte por el abandono de otros agentes o actores que no cumplen con sus obligaciones, como los entes públicos. Lo que se ha encontrado en este caso es que la empresa ha hecho inversión social en la primera etapa del proyecto y la comunidad quisiera que ese apoyo se mantuviera en el tiempo. 
Ahora bien, la cuestión estaría en que ese apoyo no derive en una relación paternalista en la que la comunidad sólo espera recibir beneficios, sin ningún tipo de contraprestación. De aquí se deduce que, como en muchos otros casos, los diferentes actores involucrados no entienden la responsabilidad empresarial de la misma manera (Scandelius y Cohen, 2016). Para los miembros de la comunidad, puede ser vista como un apoyo permanente. Para la organización, el apoyo puede variar en el tiempo, ya sea a medida que se madura la relación o que la empresa se vuelve parte de la comunidad después de operar en una determinada zona geográfica, es decir, cuando deja de ser vista como agente externo.

De cara al objetivo de la investigación, relativo a cómo se ha dado la relación y cuáles fueron los aportes desde la comunicación, se encuentra que la profesional del área participó en los equipos en los que se definieron medios y orientaciones para ese relacionamiento. Se deduce que existe interés en la organización por fortalecer el apoyo que la comunicación pueda prestar, por la contratación que se anunció de un profesional experto en el tema, pero también se evidencia que esta decisión ha atendido más al crecimiento de la empresa que a una conciencia sobre el papel estratégico que la comunicación tiene en estos procesos.

Este hallazgo guarda relación con otros trabajos en los que se ha encontrado que las decisiones de mejorar o incrementar los recursos de comunicación en muchas entidades atiende más a razones de volumen (más proyectos, más comunidades, más personas para relacionarse) y menos a una conciencia sobre la relevancia que tiene la comunicación en el logro de fines corporativos en los que se involucra a los públicos de interés (Etayo y Preciado, 2008).

Puede afirmarse que parte del desánimo que manifiestan ciertos miembros de la comunidad para asistir a las reuniones tiene que ver con que no perciben resultados de tipo material al finalizar el proceso. En este aspecto es bueno hacer notar que la comunicación, y en concreto asistir a las reuniones, no debería tener una mera connotación de medio para recibir un favor o un beneficio material. Debería ser vista como una oportunidad de conocerse, discutir acerca de las preocupaciones que se tienen, enriquecer el proceso participativo y dar a conocer puntos de vista, propuestas y análisis sobre las situaciones que se van presentando.

La falta de convergencia en las respuestas asociadas al cumplimiento de los compromisos pactados con la empresa revela falta de claridad en cuanto a cuáles fueron los acuerdos a los que se llegó en un principio. Dicha ausencia de claridad puede deberse a vacíos o ruidos en el discurso de la organización, o bien, al grado de comprensión de los mensajes por parte de cada uno de los miembros de la comunidad. Cabe preguntarse, en este renglón, si en temas como el cambio de nombre de la empresa los medios seleccionados para informar fueron suficientes.

Cuando se trata de explicar si en situaciones similares a estas, las empresas se convierten en miembros de la comunidad, de la manera como lo proponen los estudios citados en este artículo, la conclusión es que los asistentes al grupo de discusión la ven como un agente que está en otro nivel, que dispone de más recursos e influencia y que, en muchos casos, es ajeno a ellos, más que uno de ellos. De igual modo, si bien esta última condición puede ser una aspiración de la entidad, es también una categoría que se alcanza con el tiempo. 
En investigaciones futuras se precisa revisar si Celsia se ha convertido en un actor relevante de la zona, así como determinar qué variables, condiciones o circunstancias llevarían a calificarle como tal. Asimismo, convendría caracterizar la responsabilidad empresarial en la etapa del proceso en la que ha pasado a generar energía.

\section{Fuentes consultadas}

Benn, S.; Renier T. y Pendleton, J. (2010). "Public Relations Leadership in Corporate Social Responsibility”. Journal of Business Ethics, núm. 96, pp. 403-423.

Celsia (2013). “Reporte integrado 2012”. Extraída el 4/X/2017 desde http://www.celsia.com/Portals/0/contenidos-celsia/sostenibilidad/pdf/reporteintegrado-2012.pdf

Chaudhri, V. y Wand, J. (2007). "Communicating Corporate Social Responsibility on the Internet. A case Study of the Top 100 Information Technology Companies in India". Management Communication Quarterly. Vol. 21, núm. 2, pp. 232-247.

Clark, C. (2000). "Differences between Public Relations and Corporate Social Responsibility: An Analysis”. Public Relations Review. Vol. 16, núm. 3, pp. 363-380.

Colinversiones (2012). “Informe de sostenibilidad 2011”. Medellín.

Coppa, M. y Sriramesh, K. (2013). "Corporate Social Responsibility among SMEs in Italy”. Public Relations Review, núm. 39, pp. 30-39. Extraída el 4/X/2017 desde https://www.researchgate.net/profile/Krishnamurthy_Sriramesh/publication/257181829_ Corporate_social_responsibility_among_SMEs_in_Italy/links/0a85e5315d954ba81b000000/ Corporate-social-responsibility-among-SMEs-in-Italy.pdf

Dawkins, J. (2004). “Corporate Responsibility: The Communication Challenge”. Journal of Communication Mangement. Vol. 9, núm. 2, pp. 108-119.

Empresa Urrá (2012). “ABC de las transferencias”. Extraída el 4/X/2017 desde http://www.urra.com.co/Transferencias/abctransferencia.pdf

Etayo, C. y Preciado, A. (2008). "La comunicación directiva interna en las agencias de publicidad españolas”. Anàlisi, núm. 37, pp. 11-29. Extraída el 4/X/2017 desde www.raco.cat/index.php/Analisi/article/download/138542/189534

Falck, O. y Heblich, S. (2007). "Corporate Social Responsibility: Doing well by doing good”. Business Horizons, núm. 50, pp. 247-254.

Freeman, R. y Phillips, R. (2002). "Stakeholder theory: A Libertarian Defense". Business Ethics Quarterly. Vol. 12, núm. 3, pp. 331-349. Extraída el 4/X/2017 desde 
https://www.researchgate.net/publication/228168804_Stakeholder_Theory_A_ Libertarian_Defense

Galeano, M. (2009). Estrategias de investigación social cualitativa. El giro en la mirada. Medellín: La Carreta Editores.

Grunig, J. (2011, septiembre 14). "The value of public relations: Relationships and reputation as an intangible assets". Conferencia brindada en el V Congreso Internacional de Comunicación Estratégica. Gestionar la confianza frente a nuevos escenarios de interrelación. Universidad del Norte, Barranquilla, Colombia.

Ihlen, O. (2008). "Mapping the Environment for Corporate Social Responsibility. Stakeholders, Publics and the Public Sphere". Corporate communications. An international Journal. Vol. 13, núm. 2, pp. 135-146.

Jung, H. y Kim, D. (2016). “Good Neighbors but Bad Employers: Two Faces of Corporate Social Responsibility Programs”. Journal of Business Ethics, núm. 138, pp. 295-310. Extraída el 4/X/2017 desde

https://www.researchgate.net/publication/276820793_Good_Neighbors_but_Bad_ Employers_Two_Faces_of_Corporate_Social_Responsibility_Programs

Leeper. K. (1996). "Public Relations Ethics and Communitarianism: A Preliminary Investigation”. Public Relations Review. Vol. 22, núm. 2, pp. 163-179.

Luning, S. (2012). "Corporate social responsibility (CSR) for exploration: Consultants, companies and communities in processes of engagements". Resources policy, núm. 37, pp. 205-211.

Moreno, M. y Capriotti, P. (2007). "Communicating Corporate Responsibility through Corporate Web Sites in Spain”. Corporate Communications: An International Journal. Vol. 12, núm. 3, pp. 221-237.

Recalde, M. (2010). “¿De qué modo contribuye la comunicación al logro de una empresa más responsable?” Cuadernos de Información, núm. 27, pp. 111-122. Extraída el 4/X/2017 desde http://cuadernos.info/index.php/CDI/article/view/27

Rodríguez-Raga, J. y Seligson, M. (2010). "Cultura política de la democracia en Colombia, 2010. Consolidación democrática en las Américas en tiempos difíciles". Extraída el 4/X/2017 desde https://www.vanderbilt.edu/lapop/colombia/Cultura_politica_ de_la_democracia_en_Colombia_2010.pdf

Scandelius, C. y Cohen, G. (2016). “Achieving collaboration with diverse stakeholders. The role of strategic ambiguity in CSR communication". Journal of Business Research, núm. 69, pp. 3487-3499. 
Self, C. (2010). "Hegel, Habermas, and Community: The Public in the New Media Era". International Journal of Strategic Communication, núm. 4, pp. 78-92.

Vaaland, T. y Heide, M. (2008). "Managing corporate social responsibility: lessons from the oil industry". Corporate Communications: An International Journal. Vol 13, núm. 2, pp. 212-225.

Wirth, H. y otros (2016). “Corporate Social Responsibility: Communication about social and environmental disclosure by large and small copper mining companies". Resources Policy, núm. 49, pp. 53-60.

Wood, D. y Logsdon, J. (2008). “Business citizenship as a metaphor and reality”. Business Ethics Quarterly. Vol. 18, núm. 1, pp. 51-59. 